\title{
Development of an advanced electropolishing setup for multicell high gradient niobium cavities
}

\author{
F. Éozénou, ${ }^{*}$ S. Chel, Y. Gasser, C. Servouin, B. Visentin, and J-P. Charrier \\ CEA, Irfu, SACM, Centre de Saclay, F-91191 Gif-Sur-Yvette, France \\ Z. Wang \\ ENSAM ParisTech, F-75013 Paris, France \\ (Received 28 March 2012; published 3 August 2012)
}

\begin{abstract}
Reproducible operation at high performances of superconducting cavities is required for linear accelerators. High beta elliptical cavities are thus of concern and, to achieve required performances for such resonators, surface preparation including electropolishing is recommended. We have designed and operate a setup for electropolishing in the vertical position of multicell cavities in order to: (i) obtain high yield with large elliptical cavities for Superconducting Linac (SPL) or European Spallation Source projects; (ii) develop a reference installation demonstrating that this process is appropriate for the large scale treatment of cavities in industry. The setup described here is the first one able to electropolish vertically multicell cavities with circulating acid and high safety standards. This equipment makes it possible to use a wide range of parameters such as voltage, acid flow rate, temperature, and nitrogen injection with an R\&D purpose in mind. Optimization is studied using modeling with COMSOL software for different cavities. As examples, we present some results for the $704 \mathrm{MHz}$ high-beta SPL cavity and the $1300 \mathrm{MHz}$ International Linear Collider cavity and show the influence of cathode shape on both acid flow and electric field distribution during the process. Importance of the size of the cavity and first results achieved on single-cell and nine-cell cavities will be discussed.
\end{abstract}

DOI: 10.1103/PhysRevSTAB.15.083501

\section{INTRODUCTION}

Electropolishing (EP) is believed to be the most desirable treatment for SRF cavities [1]. EP is an anodic electrochemical treatment carried out in concentrated hydrofluoric-sulfuric $\left(\mathrm{HF}-\mathrm{H}_{2} \mathrm{SO}_{4}\right)$ acids. Generally, the cavity is electropolished following a process developed by KEK [1]: A voltage is applied between the cavity and a coaxial aluminum rod cathode while the cavity is set in horizontal position, rotating, and half-filled with the circulating acid. This process makes it possible to reach high gradients on nine-cell elliptical Tesla-shape cavities for electrons and has been chosen for the surface treatment of cavities for the European X-ray Free Electron Laser linac. Large scale cavity treatment has been successfully carried out in different institutes [2,3]. However, it induces some drawbacks: the rotating seals are more easily prone to acid leaks, the cavity must be switched full of acid for draining, and the footprint of the setup is rather large. Furthermore, the niobium removal rate in the cavity depends on the location of the cell [4]. To overcome these drawbacks, some laboratories are investigating an alternate process where the cavity is electropolished in the vertical

\footnotetext{
*fabien.eozenou@cea.fr
}

Published by the American Physical Society under the terms of the Creative Commons Attribution 3.0 License. Further distribution of this work must maintain attribution to the author(s) and the published article's title, journal citation, and DOI.
PACS numbers: 74.70.Ad, 81.65.Ps, 82.47.Wx, 82.45.Qr

position (VEP) [5]. Cornell University has proved that VEP with static acid could make it possible to reach high gradients [6], and as VEP is suitable to the treatment of large elliptical cavities, we have developed at CEA-Saclay a VEP setup sized for the largest high beta and high gradient cavity we have designed, a $704 \mathrm{MHz}$ Superconducting Linac (SPL) cavity. All other "smaller" cavities, as International Linear Collider (ILC) cavities, fit into the setup too.

In this paper, this VEP setup is described as well as results concerning the modeling of VEP for both high beta SPL [7] and ILC cavities. The acid flow and electrical field distribution expected during VEP will be described, as well as first results achieved on single-cell cavities.

\section{AN ADVANCED SETUP}

\section{A. Generalities}

This setup (see Fig. 1) is designed to electropolish a cavity in the presence of circulating acid electrolyte $(300 \mathrm{~L}$ storage capacity). The expected acid flow is between 5 and $40 \mathrm{~L} / \mathrm{min}$. An EP process using a constant voltage has been chosen for the treatment of the cavities. The available electric power for the electropolishing is $30 \mathrm{~kW}(20$ $\mathrm{V}-1500 \mathrm{~A})$. The setup is confined in two distinct ventilated cabinets, where as a single-booth setup is used in another institute [8]. In our case, the cavity (located in the main cabinet) is filled from the bottom thanks to a membrane pump (located in the pit) and the acid goes back to the 


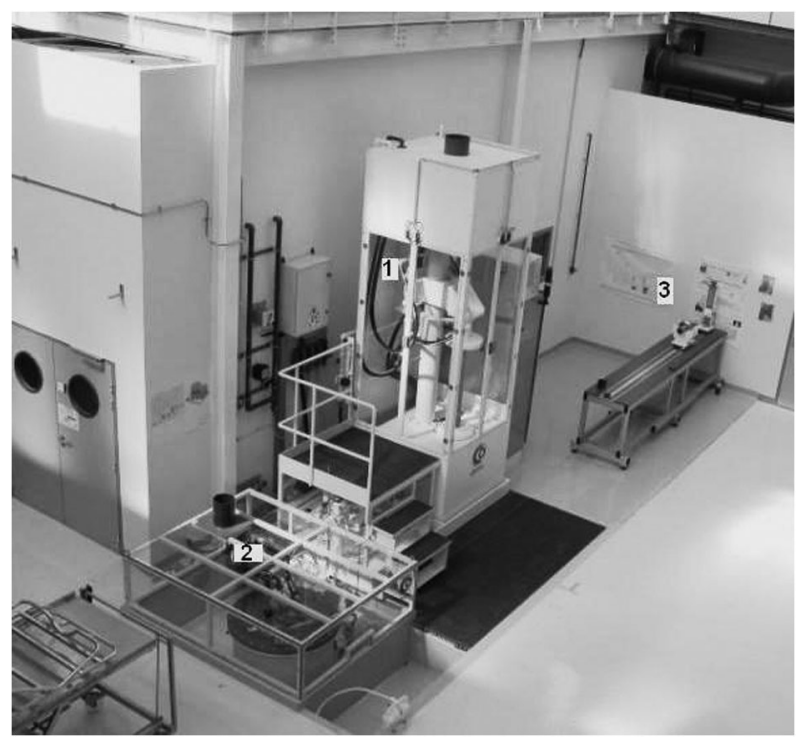

FIG. 1. VEP setup at CEA Saclay. 1: Main cabinet for cavity treatment. 2: Acid tank located in a pit. 3: Table for assembly prior to VEP.

tank thanks to gravity. At the end of an electropolishing sequence, the acid is drained back to the tank and the cavity is rinsed with ultrapure water. A predetermined sequence of filling (from the bottom)/draining of the cavity is followed by a continuous circulating sequence. Rinsing stops automatically as soon as water conductivity is low enough.

We intended to benefit from our experience in horizontal EP [9] to run an operator friendly system with substantial improvements: (i) simplified handling of the cavity and the cathode; (ii) low floor surface; (iii) simplified emptying of the cavity and improved rinsing procedures; and (iv) fully automated process, run through a touchscreen (Fig. 2).

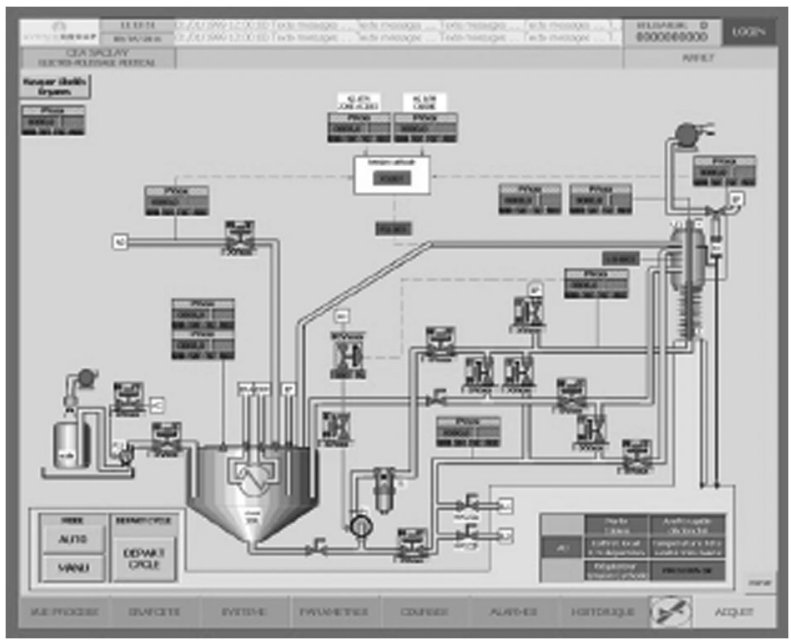

FIG. 2. Flowsheet of the process displayed on the touchscreen.
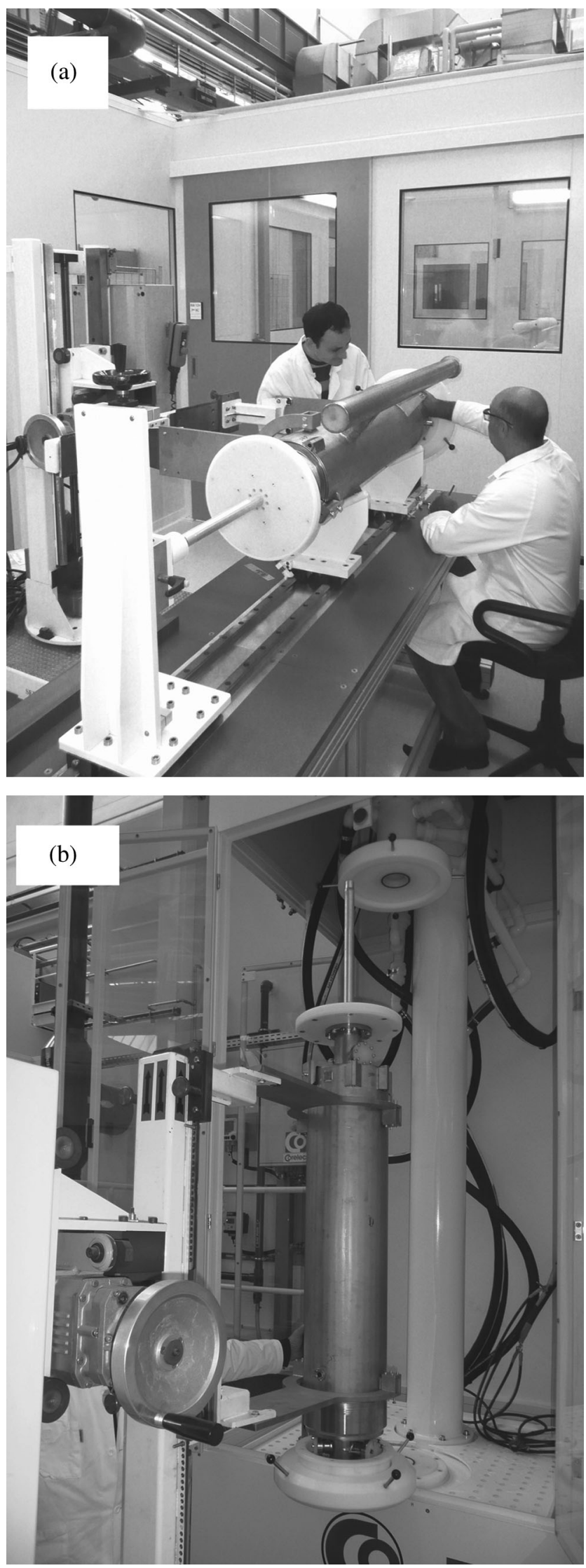

FIG. 3. (a) Insertion of the cathode in the cavity prior to (b) transfer into the EP setup. 
The insertion of the cathode into the cavity and assembly of connecting pieces are done on a dedicated table close to the setup [Figs. 1 and 3(a)]. The cavity is then transferred into the main cabinet with a specific handling tool [Fig. 3(b)].

\section{B. Safety}

Safety aspects have been carefully taken into consideration to design the setup. Nitrogen is blown on the top of the cavity and on the top of the acid tank to dilute the hydrogen generated during the process and prevent any risk of explosion. Furthermore, the program of the automat includes specific procedures if sensors (temperature, pressure, hydrogen, flows, acid level, intensity, and voltage) should detect any failure.

Fundamental parameters (temperature, liquid flow, voltage) evolution is monitored on the touchscreen of the automat, as well as failure occurrence. A USB port allows an easy exportation of data.

\section{Materials}

The inner part of the acid tank is coated with Teflon and piping/valves are made of perfluoroalkoxy. Polyvinylidene fluoride has been chosen for the pieces requiring higher mechanical resistance (connections for the cavity, inserts for sensors).

A heat exchanger made of Teflon is inserted in the acid tank for temperature control of the cavity $(18 \mathrm{~kW}$ capacity).

\section{PARAMETERS TO OPTIMIZE}

\section{A. A process dominated by $\boldsymbol{F}^{-}$ion diffusion}

The described setup was designed with $R \& D$ purpose in mind, with the view of tracking the optimal parameters for VEP process. Experiments on samples and on cavities were carried out in different institutes for precise understanding of the niobium electropolishing process [10-13].

The studies using rotating disk electrodes (RDE) and electrochemical impedance spectroscopy measurements [11-13] have demonstrated the dominant role of fluorine diffusion as a limiting electrochemical step in the presence of a surface film considered as a salt film [11] or porous oxide [13]. As a result, current density will be dominated by the concentration gradient in the $F^{-}$ion through the diffusion layer once the niobium surface temperature is controlled.

\section{B. Influence of fluid dynamics}

The EP process is then impacted by fluid dynamics: (i) A poor $\mathrm{HF}$ renewal at the cavity surface will be responsible for a decrease in current and surface deterioration [10,14]. (ii) A low fluid velocity close to the cell will be responsible for a thicker diffusion layer and lower concentration gradient. (iii) Increased flow will then promote the forming of

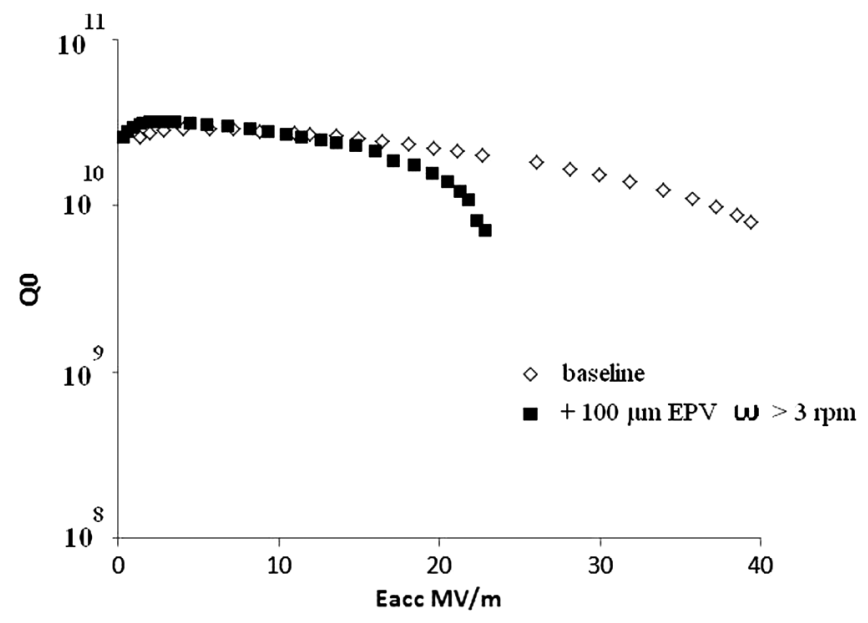

FIG. 4. Deterioration of C1-21 single-cell cavity after horizontal $\mathrm{EP}$ at rotation speed $\omega>3 \mathrm{rpm}$.

the diffusive layer. (iv) Too high fluid turbulences should be responsible for a deterioration of the viscous layer forming at the niobium surface during EP.

In horizontal configuration, $1 \mathrm{rpm}$ cavity rotation speed and $10 \mathrm{~L} / \mathrm{min}$ acid flow are commonly applied although this set of parameters although it is not optimal in terms of the diffusion plateau [15]. A single-cell cavity (C1-21) was horizontally electropolished at high rotation speeds ( $3 \mathrm{rpm}<\omega<6 \mathrm{rpm}$ ) and tested to investigate the impact of excessive fluid velocity. A deteriorated surface finishing was observed after $100 \mu \mathrm{m}$ removal and the accelerating gradient decreased from 39 to $23 \mathrm{MV} / \mathrm{m}$ (Fig. 4).

To avoid such a disagreement, an optimal fluid configuration has then to be established in vertical configuration.

\section{Cavity EP and electromigration}

Furthermore, the nonuniformity of the removal in a cell (at least twice as high at the iris compared to equator) suggests that the influence of electromigration has also to be considered at the cavity scale. Improvement of the electric field under applied voltage has been taken into account by researchers for optimization [16-19].

\section{MODELING OF VERTICAL EP}

The use of modeling is a powerful tool, complementary to studies carried out on samples. Chemical treatments applied to niobium cavities have motivated researchers to numerically investigate operating parameters (fluids, electric field, concentrations, thermal properties, etc.) [16-21]. The considerations above have motivated us to model both fluid dynamics and electric field, considered as static, during VEP in different configurations.

\section{A. 2D axisymmetry model with COMSOL multiphysics}

In this paper, fluid distribution and electric field have been modeled for SPL and ILC cavities with a cylindrical 


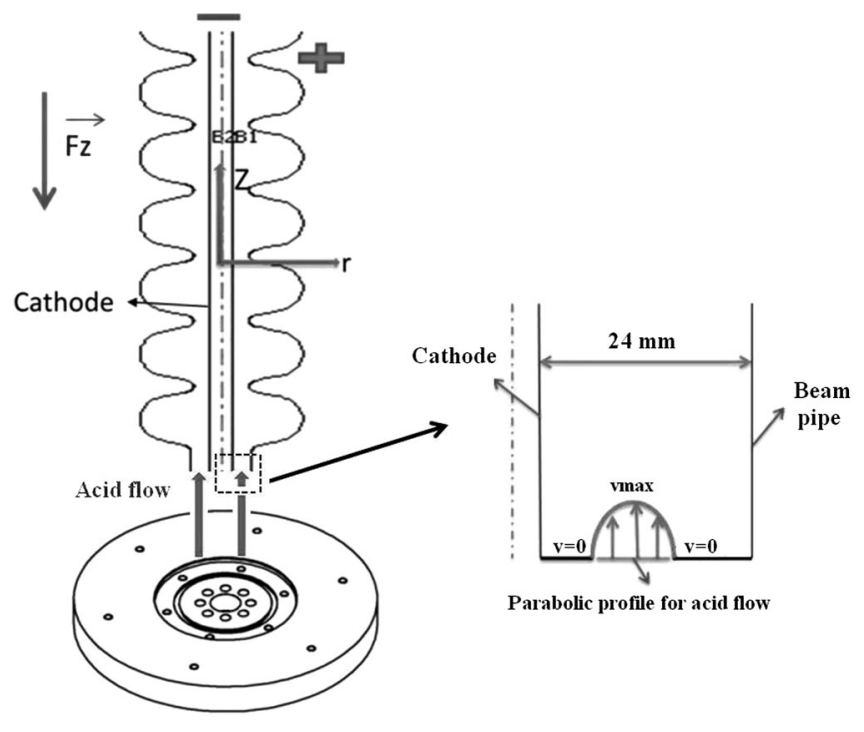

FIG. 5. 2D axisymmetry used for modeling of VEP. We have considered a crown of fluid entering the cavity with a parabolic profile.

cathode (30 mm diameter). In a second step, alternative cathode shapes have been numerically investigated for possible improvement of the process.

Contrary to horizontal EP, VEP is a symmetrical geometry to model. Gravity force, represented by Fz on Fig. 5, does not depend on a radial component. It is thus possible to create an axisymmetric 2D model according to the cavity $z$ axis (see Fig. 5) which makes the calculation easier compared to a 3D model.

\section{B. Fluid dynamics modeling}

For the considered setup, acid is introduced into the cavity through eight holes equidistant from the cathode. To simplify the model, a crown of acid, with parabolic profile, has been considered, as described in Fig. 5. The maximum velocity in the middle of the parabola is called vmax.

Fluid velocities below $0.8 \mathrm{~m} / \mathrm{s}$ were used in the model. As a consequence, Navier-Stokes equations, related to laminar flow of incompressible fluid were chosen to model the fluid dynamics in the cavity:

$$
\begin{gathered}
\rho \frac{\partial u}{\partial t}-\eta \nabla^{2} u+\rho(u \cdot \nabla) u+\nabla p=G \\
\nabla \cdot u=0 .
\end{gathered}
$$

$\eta$ is the dynamic viscosity $(\mathrm{Pa} \cdot \mathrm{s}) ; u$ the fluid velocity $(\mathrm{m} / \mathrm{s}) ; p$ the pressure $\left(\mathrm{N} / \mathrm{m}^{2}\right) ; G\left(\mathrm{~N} / \mathrm{m}^{3}\right)$, the resultant force, only the gravity is considered here.

According to the boundary conditions, fluid neither slips at the cathode nor at the cavity surface.

\section{Electric field modeling}

For the electric field modeling, a potential equal to zero has been chosen for the cathode and a $15 \mathrm{~V}$ potential has been applied to the cavity. Only primary current distribution is considered in the modeling: the voltage drop in the anodic layer is assumed homogeneous along the cavity and the motion of the ions (Nernst-Planck equations) is not taken into account. This model is simplified but should give a good hint towards an improved configuration. To achieve better homogeneity, some parts of the cathodes have been insulated as explained in the following paragraphs.

\section{FLUID DYNAMICS: VEP VS HORIZONTAL EP}

Unlike the process investigated in the present paper, a hollow cathode is used for horizontal EP. It enables one to inject the acid into the cavity through holes located in front of the equators. Previous models [17] have shown that such a configuration is inappropriate for homogenous fluid distribution. In fact, fluid velocity increases from the center of the cavity to extremities. This inhomogeneity might be responsible for the degradation of field flatness observed after the horizontal EP of nine-cell cavities [4]. A similar investigation has been carried out for the VEP of nine-cell cavities with a cylindrical cathode.

Results obtained with a rod cathode and vmax $=$ $0.2 \mathrm{~m} / \mathrm{s}$ are shown on Fig. 6. Along each line 1-5 considered in the central cell, the fluid profile is similar to the shape shown in Fig. 6(c).

This simulation highlights that, in the VEP case, fluid distribution does not depend on the position of the cell in the cavity. The graph shows a fluid profile with two distinct parts: the fluid flows principally in the continuation of the beam pipe [see Fig. 6(c)], and the second bump characteristic of the flow in the cell is lower $(10 \%$ of the maximum flow). A slight asymmetry in each cell is also suggested, with higher flow in the upper half cell. It is then necessary to modify the geometry to improve the expected removal rate of niobium. It has been proved that shaped cathodes make it possible to improve the process [16-19]. With this purpose in mind, different cathode shapes were modeled in our configuration. They are aimed at generating obstacles to guide the acid flow towards the cavity surface.

Four alternative cathode shapes were investigated for more homogeneous fluid velocity in cells (Fig. 7). In each case, fluid velocity was studied at five different locations of the central cell of the cavity.

From the analysis of related data, we have concluded: (i) The shape D allows a 39\% increase of fluid velocity close to the cell. (ii) Uniformity of fluid velocity is improved by $33 \%$ using shape C.

These two shapes are promising candidates to be tested during VEP. 


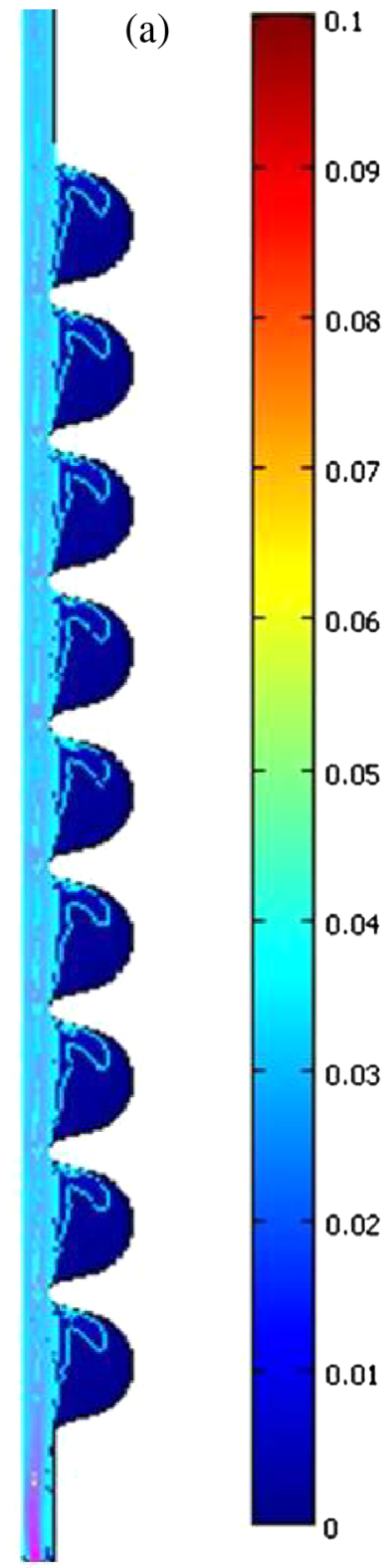

Fluid velocity $(\mathrm{m} / \mathrm{s})$
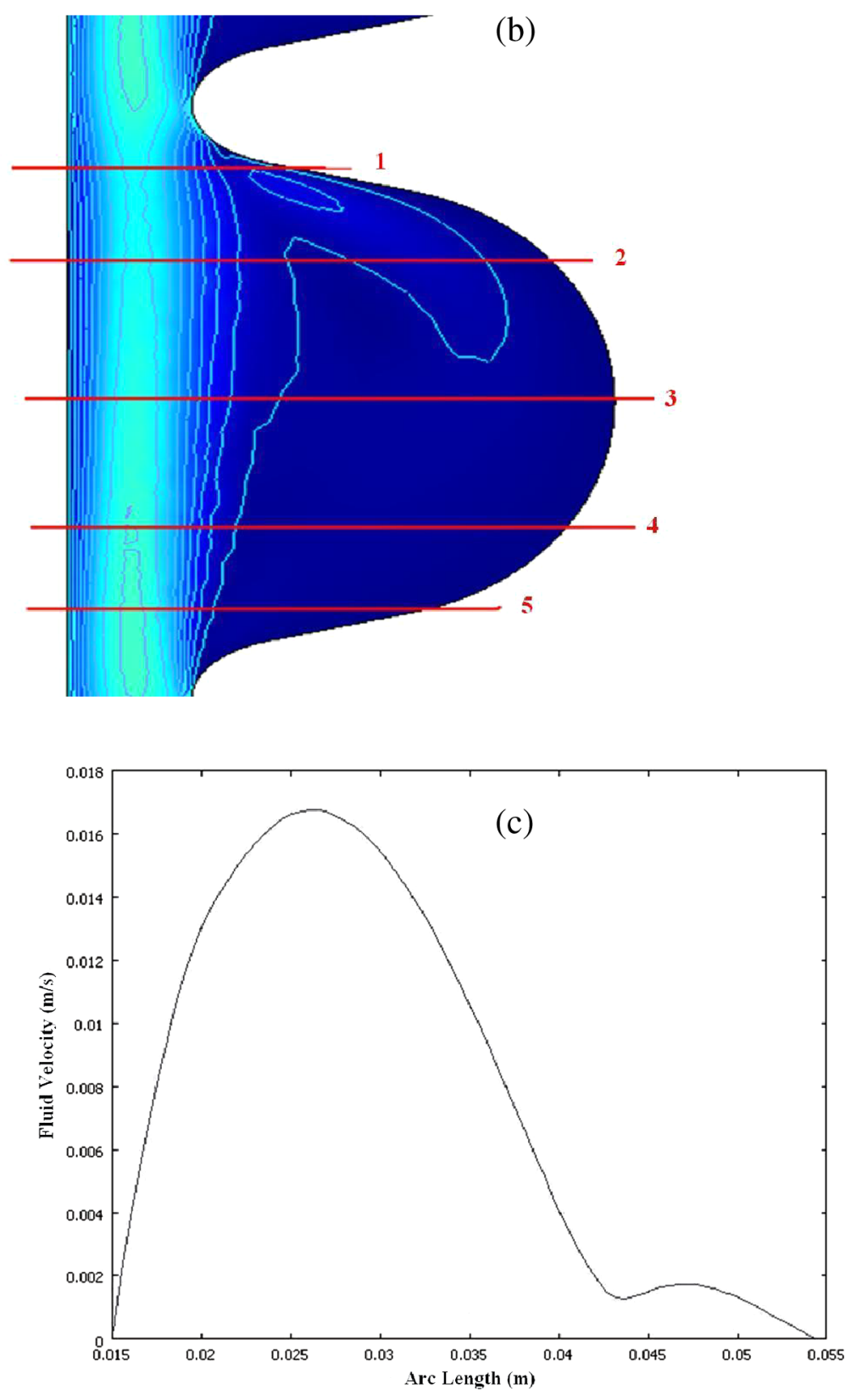

FIG. 6. (a) Fluid modeling in the ILC cavity for vmax $=0.2 \mathrm{~m} / \mathrm{s}$. (b) Zoom of the central cell. (c) For each line (1 to 5), the distribution profile between the cathode and the cavity wall is similar.
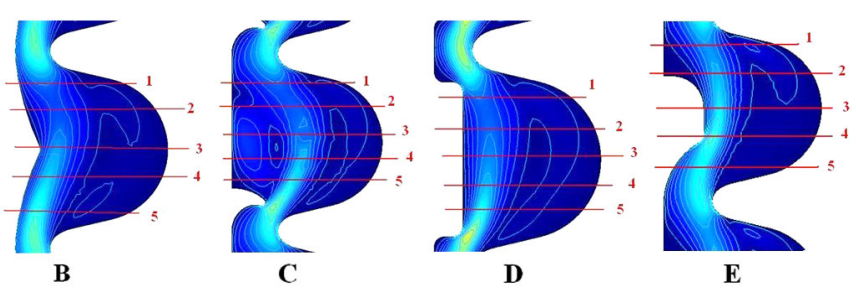

$\mathbf{E}$

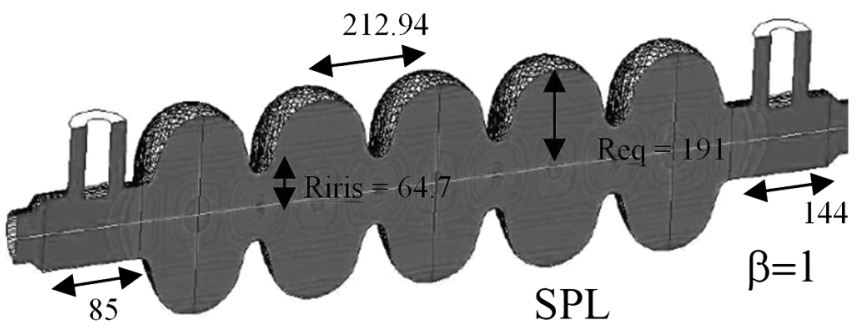

FIG. 7. Shapes tested to improve fluid distribution inside the cells.

FIG. 8. $704 \mathrm{MHz}$ SPL cavity to be electropolished in the VEP setup. 

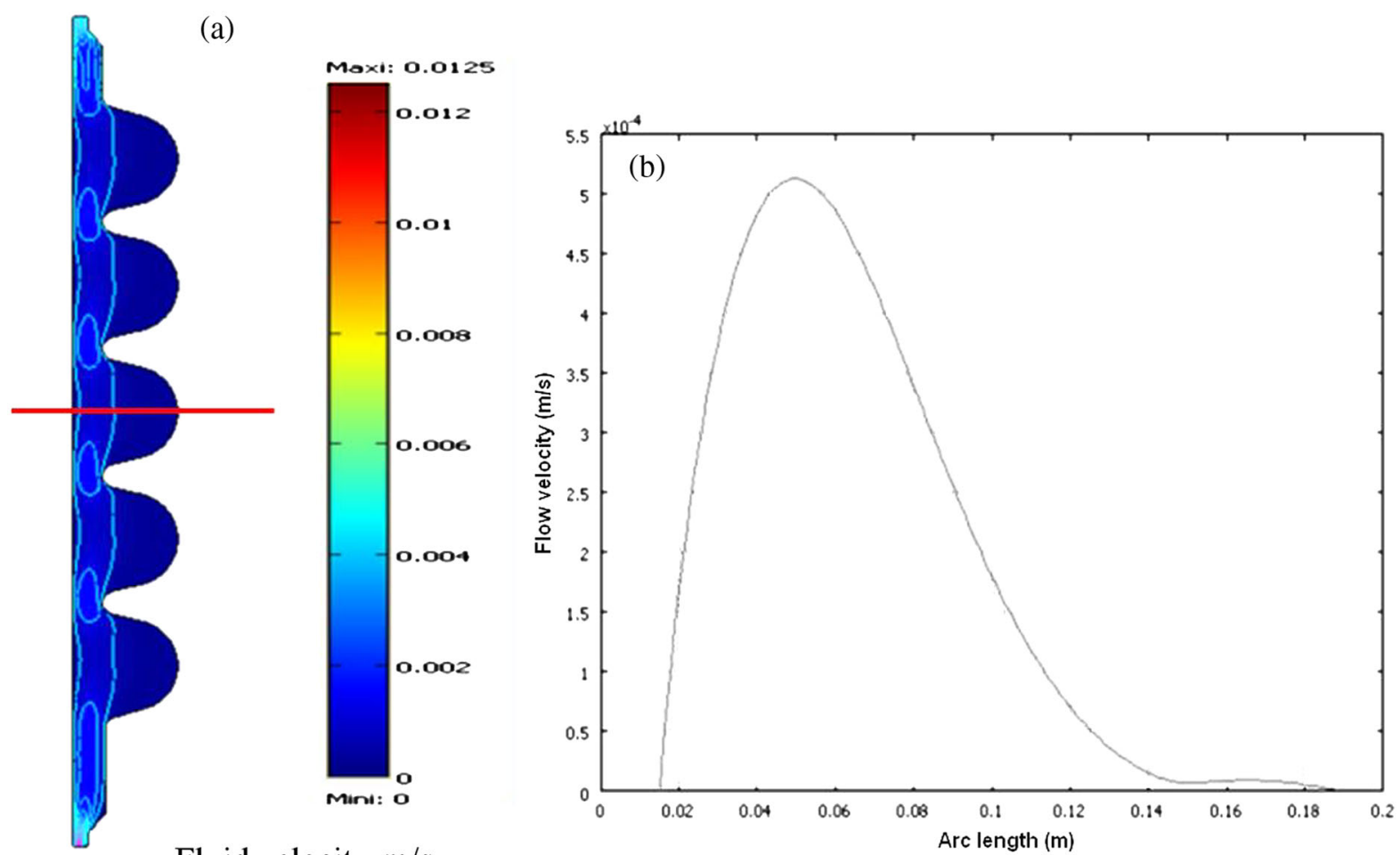

Fluid velocity $\mathrm{m} / \mathrm{s}$

FIG. 9. (a) Fluid distribution in the five-cell SPL cavity and (b) fluid velocity profile along the red line.
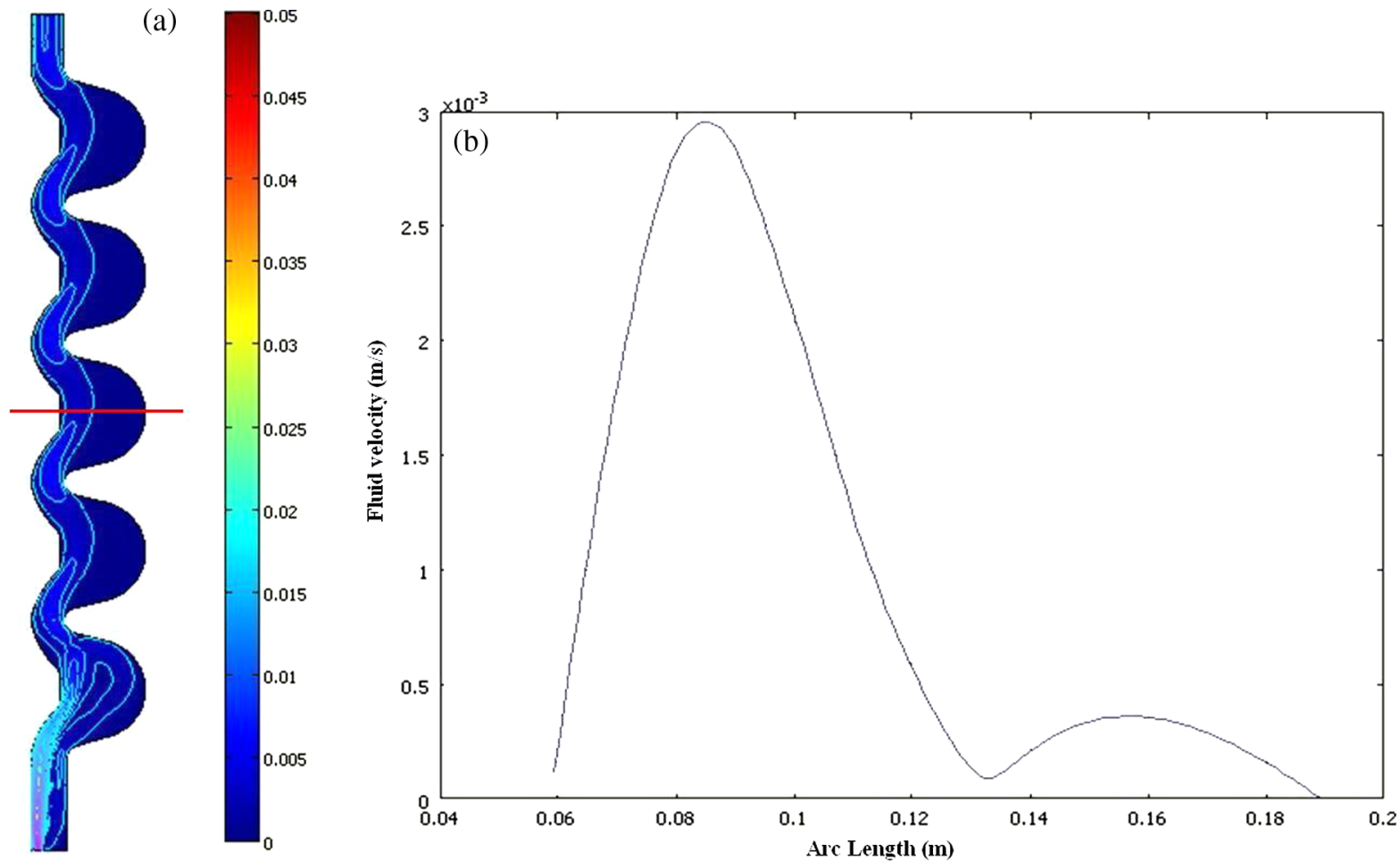

Fluid velocity $(\mathrm{m} / \mathrm{s})$

FIG. 10. (a) Fluid distribution in the five-cell SPL cavity and (b) fluid velocity profile along the line. The shaped cathode makes it possible to increase fluid velocity in the cell. 


\section{INFLUENCE OF THE SIZE OF THE CAVITY}

The experiments have highlighted fluid distribution for the Tesla-shape nine-cell cavities. As the setup is mainly dedicated to two types of cavity, it is vital to anticipate the effect of polishing parameters on the larger five-cell SPL cavity whose drawing, including characteristic dimensions, is shown in Fig. 8.

Similar equations and boundary conditions have been chosen to model fluid dynamics with a cylindrical cathode. Fluid distribution in such a configuration is represented in Fig. 9, with $\operatorname{vmax}=0.8 \mathrm{~m} / \mathrm{s}$. The shorter beam pipe is located on top.

We notice that fluid velocity at the cavity surface is very low. The fluid distribution along the horizontal line shows that the velocity in the cell is negligible compared to the velocity at the cathode surface.

These results show that EP is less adapted for larger cavities. In fact, stagnant acid in the cell is undesirable because of: (i) low convection at the cathode surface; (ii) local saturation of the acid in niobium.

It is then vital to anticipate more adapted cathode geometries. Similarly to the ILC cavity case, an alternative shape was also designed for the SPL cavity. We have considered a beam pipe without taper at the extremities to allow the use of a cathode with large protuberances. The fluid distribution achieved with this cathode shape (called F) is shown on Fig. 10. The narrowed section available for the fluid circulation increases the fluid velocity in the proximity to the cathode (flow roughly multiplied by 5.5 ) and in cells (multiplied by 5 ).

In that way, shaped cavities are desirable for VEP treatment. We also have to keep in mind that presented models do not take into account the forming of gases during the process. Hydrogen forming at the cathode might have an impact on fluid mixing. However, observations during EP of samples using a vertical bar cathode, and the shape of the modeled flow in cavities (velocity is at least 1 order of magnitude higher in the continuation of the beam pipe compared to the cell), suggest that hydrogen bubbles should be evacuated in a close volume around the cathode and should not alter modeling inside cells. It is confirmed by first sequences with both single-cell and nine-cell cavities, since no obvious trace of bubble is visible at the cavity surface when adequate voltage is applied. A too high voltage will generate oxygen at the cavity surface. These aspects will be discussed later.

\section{STUDY OF THE ELECTRIC FIELD}

Models described in previous paragraphs show that uniformity of the acid flow inside cells might be improved by using a shaped cathode. Furthermore, removal rate during EP also depends on electromigration of ions. Thus, a uniform electric field during EP along the cells is desirable for uniform removal of the surface.

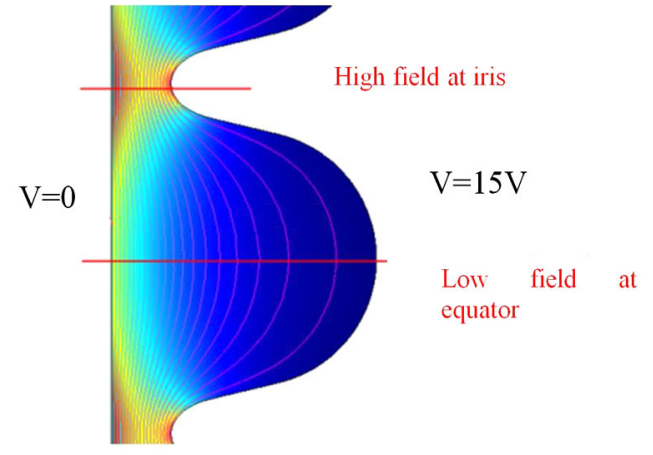

FIG. 11. Potential distribution in a Tesla-shape cell using a cylindrical cathode. At the cathode $\mathrm{V}=0$ and at the anode $\mathrm{V}=15 \mathrm{~V}$.

The electric field during EP using a cylindrical cathode has been modeled in the cells of ILC cavities. A voltage of $15 \mathrm{~V}$ between the cavity and the cathode has been used for calculations. Figure 11 below shows the isopotential curves in a cell. Close to the iris, short intervals are observed between the lines, generating a high electric field $E_{i}$ : $825 \mathrm{~V} / \mathrm{m}$. At the equator, the field $E_{e}$ is divided by more than 40: $20 \mathrm{~V} / \mathrm{m}$.

Thanks to the protuberances investigated in the previous paragraphs, the average cavity-cathode distance is more homogenous, favorable for a more uniform removal rate along the cell. In a second step, insulation of the parts of the cathode in front of the iris allows additional improvement of the uniformity. In order to evaluate quantitatively

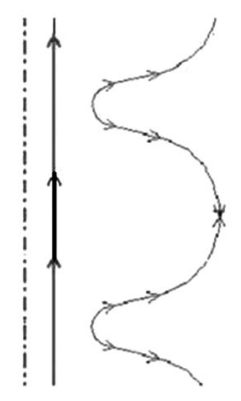

A

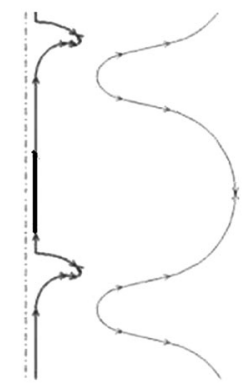

B

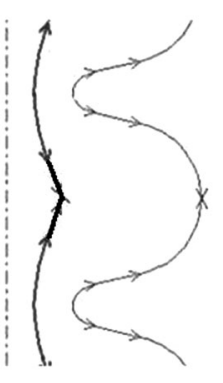

C

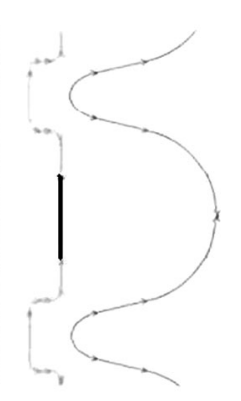

D

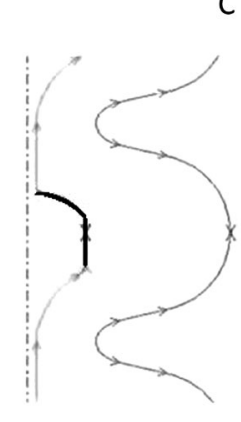

$E$
FIG. 12. Alternative insulated cathode shapes numerically tested for the Tesla cavity. At the cavity $\mathrm{V}=15 \mathrm{~V}$ and at the cathode $\mathrm{V}=0$. The active part of each cathode (dark color) is located in front of the equator. 
TABLE I. Electric field at the iris and at the equator of the ILC cavity for different cathode configurations.

\begin{tabular}{lcccc}
\hline \hline Shape & Insulation & $E_{i}(\mathrm{~V} / \mathrm{m})$ & $E_{e}(\mathrm{~V} / \mathrm{m})$ & $E_{i} / E_{e}$ \\
\hline A & No & 825 & 20 & 41.2 \\
B & No & 816 & 18 & 45.3 \\
C & No & 913 & 33 & 27.7 \\
D & No & 1138 & 43 & 26.5 \\
E & No & 600 & 35 & 17.1 \\
A & Yes & 200 & 50 & 4.0 \\
B & Yes & 96 & 15 & 6.4 \\
C & Yes & 225 & 30 & 7.5 \\
D & Yes & 128 & 35 & $\mathbf{3 . 7}$ \\
E & Yes & 240 & 32 & 7.5 \\
\hline \hline
\end{tabular}

the benefits of the investigated cathodes, the electric field has been modeled for both nine-cell and five-cell cavities, in different configurations (as an example, Fig. 12 shows configurations used for the nine-cell cavity): (i) cylindrical cathode (shape A), without insulation; (ii) cylindrical cathode with insulation at the iris; (iii) shaped cathodes (shapes B, C, D, E, F) without insulation; (iv) shaped cathode with insulation

Results (voltage of $15 \mathrm{~V}$ between the cavity and the cathode) are summed up in the recapitulative Table I.

Similar investigation has been carried out for the SPL five-cell cavity with a cylindrical cathode and the shaped one shown on Fig. 10. Thanks to shaping and insulation the ration $E_{i} / E_{e}$ improved from 36.1 to 5.9.

For both ILC and SPL cavities, designed cathodes make it possible to improve electric field uniformity in the cells. However, we have to keep in mind that an insulated cathode will be responsible for a decreased removal rate.

\section{FIRST RESULTS: INFLUENCE OF VOLTAGE, ACID FLOW, AND TEMPERATURE}

The first electropolishing in the vertical position (EPV) treatments with acid are carried out with single-cell and nine-cell Tesla-shape cavities. In spite of the small size of single-cell cavities, the gravity is sufficient for adequate flow from the head of the cavity to the storage tank in the presence of nitrogen blowing. The process is stable (see Fig. 13) and the rinsing procedure subsequently improved compared to the EP with horizontal single-cell setup.

We thus demonstrate that the designed installation is safe, easy to handle, and could be operated easily in an industrial context. First results achieved are discussed below.

\section{A. Importance of voltage}

Presented modeling shows that the cavity geometry is prevailing in polishing effectiveness and VEP should be less favorable for larger cavities. However, the models do not take into account chemical reactions and gas forming at the niobium surface.

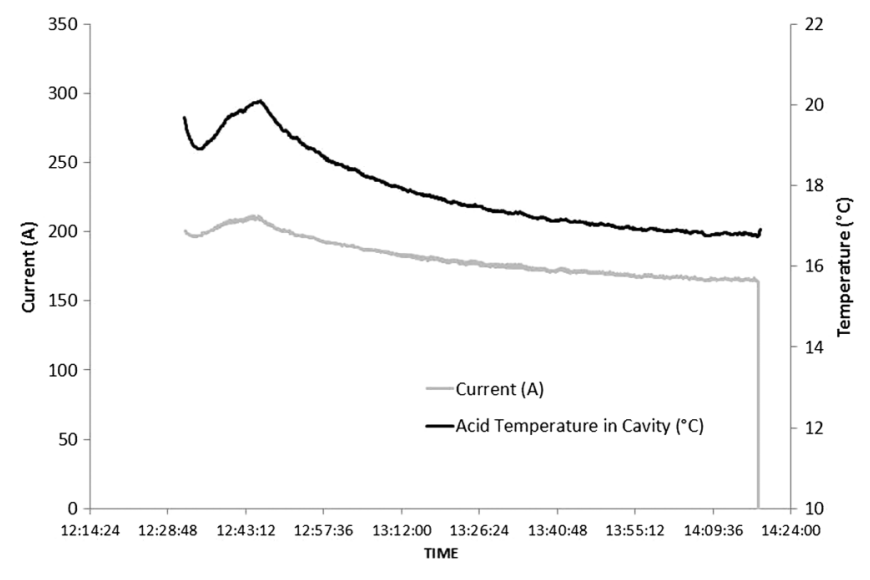

FIG. 13. Current and acid temperature as a function of time during VEP of a nine-cell Tesla-shape cavity.

Experiments on niobium samples have demonstrated that high voltage EP makes it possible to quickly achieve high brightness of the surface [22]. First experiments on single-cell cavities were carried out at $20 \mathrm{~V}$, with a shaped (simple design inspired by the shape D shown on Figs. 7 and 12) and insulated cathode at $20 \mathrm{~V}$. This cathode is shown on Fig. 14.

The electrolyte used is the standard mixture made of sulfuric acid $95 \%$ and hydrofluoric acid $40 \%$ with the ratio $9-1$. The acid temperature was below $25^{\circ} \mathrm{C}$. Acid flow was set at $8 \mathrm{~L} / \mathrm{min}$. The intensity-voltage $\mathrm{I}(\mathrm{V})$ plot shows a positive $d i / d v$ between 15 and $21 \mathrm{~V}$ (Fig. 15) indicating that oxygen is generated.

An oscillating current was observed using this set of parameters (Fig. 16). Such oscillations have already been observed in similar configuration [23], but with higher frequency. Such oscillations are associated to the brightening of the surface. It was visually confirmed: after a short

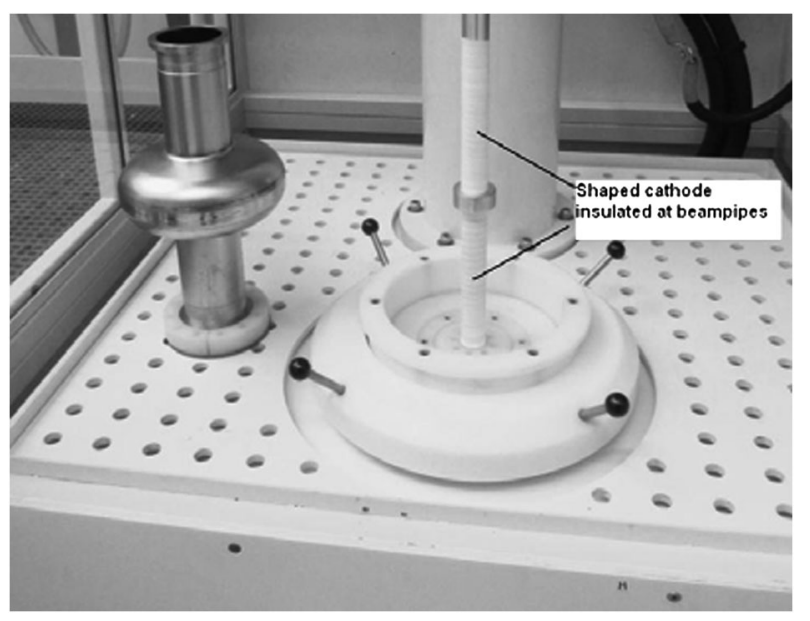

FIG. 14. Single-cell C1-21 Tesla-shape cavity and cathode used for the first VEP treatment. 


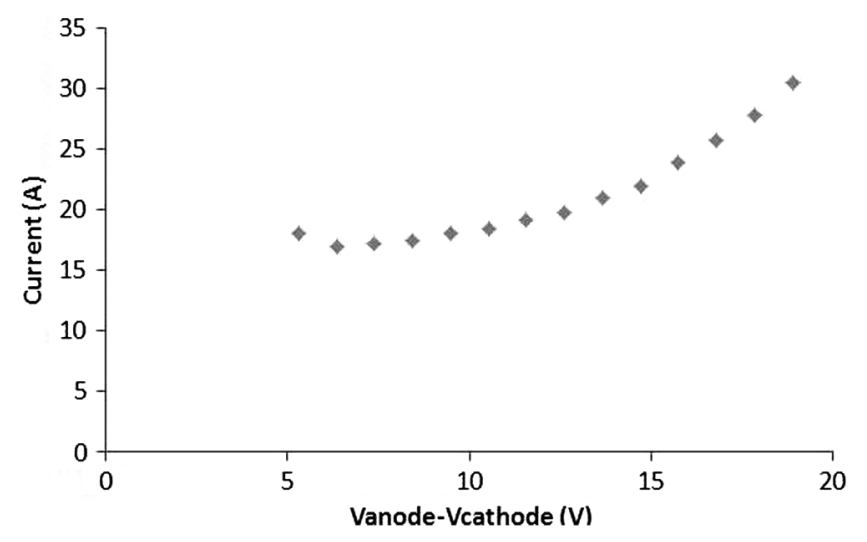

FIG. 15. I(V) curve obtained on single-cell cavity.

VEP sequence $(50 \mu \mathrm{m})$, we have improved the surface, achieving a shiny aspect. Precise surface inspection has not been carried out yet to favor rf testing of the cavity.

However, we notice a difference between the two half cells. The upper one is brighter with fine stripes resulting from the sliding of the bubbles along the viscous layer forming at the surface in presence of voltage (Fig. 17).

The cavity was rinsed with ultrapure high pressure water (HPR), assembled and baked in the cleanroom under argon atmosphere $\left(145^{\circ} \mathrm{C}-3 \mathrm{~h}\right)$ according to [24]. It was tested at $1.4 \mathrm{~K}$ and improved from its previous result to reach $30 \mathrm{MV} / \mathrm{m}$ (Fig. 18). We believe that these fine stripes do not alter the cavity performance since it was improved. Additional VEP sequences will be carried out on the same cavity.

Lower voltages have also been investigated. Electropolishing at $5 \mathrm{~V}$ was successfully tested with horizontal setup [25]. In fact sulfur is known to be generated during EP [26-28] and a lower voltage is more desirable to avoid sulfur generation at high overpotential due to

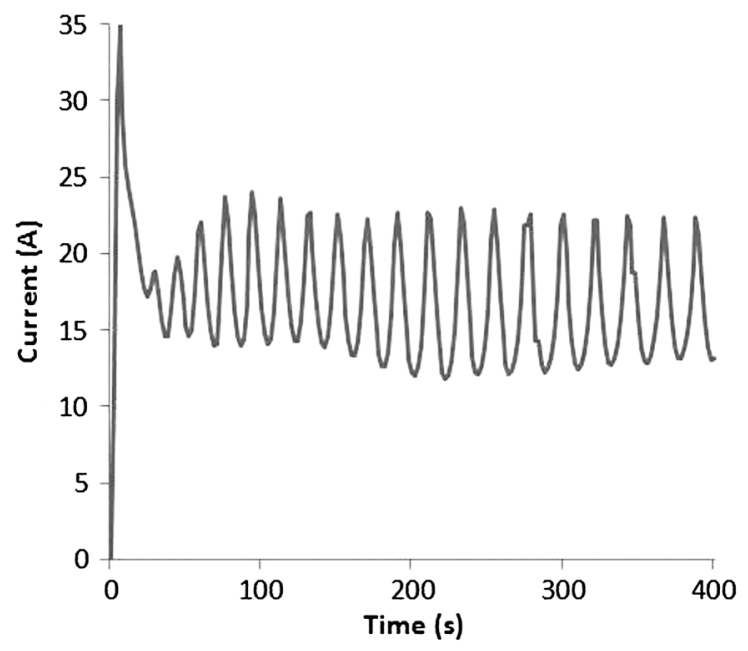

FIG. 16. Current oscillations observed during VEP of singlecell cavity at $20 \mathrm{~V}$.

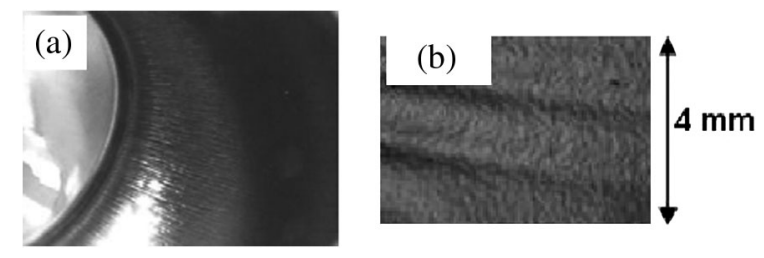

FIG. 17. (a) Bubble traces visible on the upper cell after EP at $20 \mathrm{~V}$ and (b) similar features obtained on samples electropolished vertically at similar voltage [10].

cathodic reaction [13]. Vertical EP was carried out at $10 \mathrm{~V}$. Brightening of the surface has been achieved after $10 \mu \mathrm{m}$ removal. No bubble traces were observed at the upper cell. In that way, a reduced voltage could prevent the stripes forming at the upper cell. The corresponding cavity will be tested after substantial additional removal at the same voltage.

\section{B. Influence of acid flow}

We discussed in Sec. III the need for sufficient fluid velocity close to the cavity. It is then relevant to investigate the influence of the acid flow during VEP: two I(V) curves have been plotted on a nine-cell cavity at two different acid flow rates to evaluate the evolution of the electropolishing characteristics. $11 \mathrm{~L} / \mathrm{min}$ and $17 \mathrm{~L} / \mathrm{min}$ flow rates have been tested. Corresponding results are plotted in Fig. 19.

As suggested previously, the increased flow rate favors polishing conditions during VEP since the length of the diffusion plateau is extended. The decrease in current between the two $\mathrm{I}(\mathrm{V})$ curves should be attributed to the aging of the bath between the experiments.

An increase in flow rate is then desirable and will then make it possible to: (i) improve the polishing efficiency; (ii) decrease the heating of the acid since the contact time of the acid in the cavity is reduced; (iii) facilitate the flow

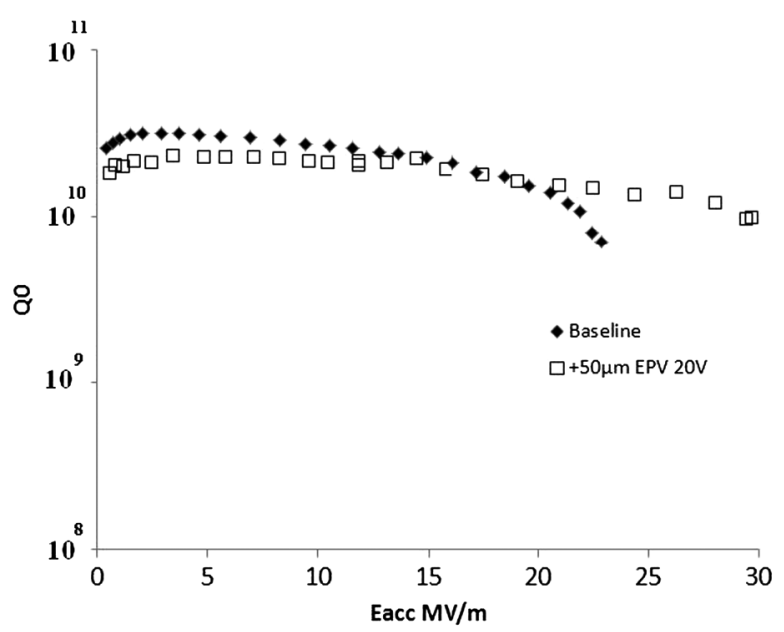

FIG. 18. Radio-frequency test of C1-21 cavity after VEP, Argon baking $\left(3 \mathrm{~h} 145^{\circ} \mathrm{C}\right)$ and HPR. 


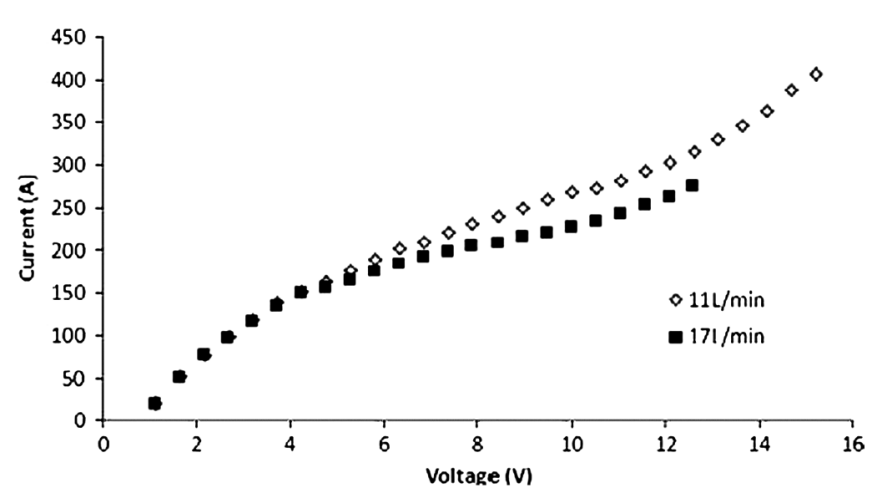

FIG. 19. Influence of acid flow on I(V) curve for nine-cell cavities. Higher flow makes the diffusion plateau more visible. Oxygen should be generated for voltage above $10 \mathrm{~V}$.

of the hydrogen bubbles and prevent them from sliding along the cells surface.

VEP has then been tested on a nine-cell dressed cavity (Fig. 3) with an acid flow of $17 \mathrm{~L} / \mathrm{min}$ at $6 \mathrm{~V}$ using a rod cathode. After 2 hours of treatment at a temperature below $20^{\circ} \mathrm{C}$, the surface has been improved (brighter surface and smoothening of grain boundaries; see Fig. 20). Only slight stripes are visible in the first cell which should be attributed to the high voltage (in the oxygen region) reached to plot the $\mathrm{I}(\mathrm{V})$ curve prior to the long VEP sequence.

Additional experiments with a nine-cell undressed cavity and a shaped/insulated cathode will follow to investigate its impact on nine-cell and compare with COMSOL models developed in Secs. V, VI, and VII.

\section{Influence of acid temperature}

VEP sequences have also been carried out on a dummy single-cell cavity at higher acid temperature, up to $35^{\circ} \mathrm{C}$. After $100 \mu \mathrm{m}$ removal we notice significant removal at the equator (approximately $60 \mu \mathrm{m}$ ). Another aspect is

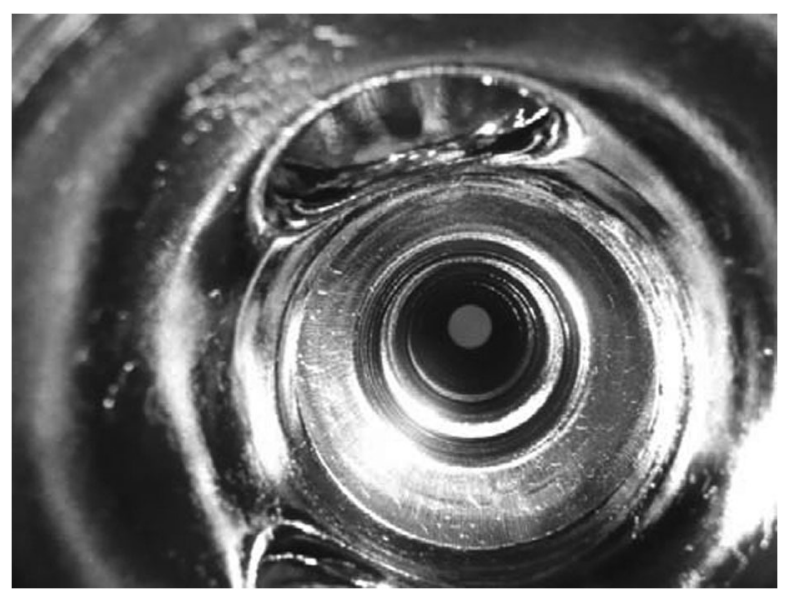

FIG. 20. Inside of a nine-cell Tesla-shape cavity (upper cells visible) after 4 hours (2 last hours at $17 \mathrm{~L} / \mathrm{min}$ ) of VEP using a rod cathode at $6 \mathrm{~V}$. noticed: a ring of pits is visible in the upper half cell, associated to a higher local removal. We assume the too high temperature is responsible for increased removal rate and local pitting of the surface. A temperature asymmetry profile has also been measured in similar configuration [29]. A local higher acid flow, as suggested from the modeling, might also favor this phenomenon. As a consequence, additional experiments will follow to determine the maximum acceptable temperature. We will also have to figure out if a two-step VEP procedure with switching of the cavity in-between is mandatory to obtain satisfactory results after long electropolishing sequences.

\section{CONCLUSION}

An advanced electropolishing tool has been developed at CEA Saclay to treat a wide range of cavities in safe and practical configuration. The treatment area and some related equipment (dedicated to cavity and cathode preparation) have been designed. Immediate feedback after cavity treatment confirms the improvements achieved.

A $30 \mathrm{MV} / \mathrm{m}$ gradient has been obtained on single-cell Tesla-shape cavity with an initial set of parameters. Modeling gives some hints to optimize the VEP for large proton cavities.

Treatments on cavities suggest that oxygen, generated at the niobium surface, is the main threat which might deteriorate the cavity surface. Acid flow rate between 11 and $17 \mathrm{~L} / \mathrm{min}$ and a low voltage (6-10 V) should be applied in order to avoid hydrogen to reach the cavity surface and generate a smooth surface. After additional tests on single-cell and nine-cell Tesla-shape cavities, ILC resonators as well as SPL and ESS prototypes cavities will be treated.

We intend to demonstrate that such a setup might be used within an industrial context for two possible midterm applications: (i) alternative to the horizontal EP for the final EP of ILC cavities; (ii) treatment of ESS (European Spallation Source) cavities.

\section{ACKNOWLEDGMENTS}

We thank the help of IPNO to carry out High Pressure Rinsing and the assistance of J-P. Poupeau, F. Ballester for the assembly of the setup, S. Berry, E. Jacques, and M. Desmons for their advice, and P. Carbonnier for the preparation of the tests of the cavities. The nine-cell cavity experiments were possible thanks to the loan of $\mathrm{C} 45$ cavity from DESY. The authors are grateful to the contribution of E. Jemy, E. Banizette, and S. Langlois to manage the safety procedures related to the EPV setup. We acknowledge the support of the European Community-Research Infrastructure Activity under the FP7 program (EuCARD, Contract No. 227579). This work has been carried out with the financial support of the "Conseil General de l'Essonne" in the frame of the ASTRE program. 
[1] K. Saito et al., in Proceedings of the 4th Workshop on RF Superconductivity, edited by Y. Kojima (National Laboratory for High-Energy Physics (KEK), Tsukuba, Japan, 1990).

[2] M. Schmökel et al., in Proceedings of the 13th International Workshop on RF Superconductivity, Beijing, China, 2007, edited by J. Hao, S. Huang, and K. Zhao (Peking University, Beijing, China, 2009), TUP31, pp. 200-203.

[3] A. Burrill et al., in Proceedings of IPAC 2011, San Sebastian, Spain (EPS-AG, Spain, 2011), MOOCA01, pp. 26.

[4] E. Kako et al., in Proceedings of the 13th International Workshop on RF Superconductivity, Beijing, China, 2007 (Ref. [2]), WEP10, pp. 453-457.

[5] R. L. Geng et al., in the 12th International Workshop on RF Superconductivity, 2005 Cornell University, Ithaca, $N Y$, USA, edited by S. Bolomestnykh, M. Liepe, and $\mathrm{H}$. Padamsee (LEPP, Cornell University, Ithaca, NY, 2007), THP04, pp. 459-463.

[6] Z.A. Conway et al., in Proceedings of the 14th International Workshop on RF Superconductivity, Berlin, Germany, 2009, edited by M. Abo-Bakr, B. Kuske, A. Liebezeit, S. Voronenko, and V. Schaa (SRF, Berlin, 2009), TUPPO004, pp. 176-179.

[7] J. Plouin et al., in Proceedings of the 15th International Workshop on RF Superconductivity, Chicago, USA, (SRF, 2011) (to be published).

[8] S. Calatroni et al., in The Proceedings of the 25th Linear Collider Conference (LINAC 2010), Tsukuba, Japan, THP032, pp. 824-826 [http://accelconf.web.cern.ch/ AccelConf/LINAC2010/papers/thp032.pdf].

[9] F. Éozénou, M. Bruchon, and J. Gantier, in Proceedings of the 13th International Workshop on RF Superconductivity, Beijing, China, 2007 (Ref. [2]), TUP80, pp. 343-346.

[10] F. Éozénou et al., in CARE Report No. 06-10-SRF, 2006, CEA, EU Contract No. RII3-CT-2003-506395.

[11] H. Tian et al., J. Electrochem. Soc. 155, D563 (2008).

[12] H. Tian and C. E Reece, Phys. Rev. ST Accel. Beams 13, 083502 (2010).

[13] F. Eozenou et al., Phys. Rev. ST Accel. Beams 13, 083501 (2010).

[14] J. Gantier, internal CEA report, 2006

[15] T. Tajima, M. P. Kelly, and J. Mammosser, in Proceedings of the 10th European Particle Accelerator Conference, Edinburgh, Scotland, 2006, edited by C. Biscari,
H. Owen, Ch. Petit-Jean-Genaz, J. Poole, and J. Thomason (EPS-AG, Edinburgh, Scotland, 2006), MOPCH179, pp. 484-486.

[16] S. Calatroni et al., in Proceedings of the 11th workshop on RF superconductivity (SRF 2003), edited by D. Proch (DESY, Hamburg, 2004).

[17] M. Bruchon et al., in Proceedings of the 13th International Workshop on RF Superconductivity, Beijing, China, 2007 (Ref. [2]), TUP51, pp. 247-250.

[18] N. Steinhau-Kühl et al., in Proceedings of the 13th International Workshop on RF Superconductivity, Beijing, China, 2007 (Ref. [2]), TUP33, pp. 204-206.

[19] A. Wu et al., in Proceedings of IPAC 2011, San Sebastian, Spain (Ref. [3]), MOPC117, pp. 352-354.

[20] C.E. Reece, in Proceedings of the 14th International Workshop on RF Superconductivity, Berlin, Germany, 2009 (Ref. [6]), THPPO061, pp. 742-745.

[21] S. Subramanian et al., in International Congress on Advanced Nuclear Power Plants, Hollywood, Florida, 2002 [http://ncacm.unlv.edu/HTML/research/nc/publication_ nc_icapp2002.pdf].

[22] F. Éozénou, M. Bruchon, and J. Gantier, in Proceedings of the 13th International Workshop on RF Superconductivity, Beijing, China, 2007 (Ref. [2]), pp. 311-317.

[23] R. L. Geng et al., in Proceedings of the 11th workshop on RF superconductivity (SRF 2003) (Ref. [16]).

[24] B. Visentin et al., in Proceedings of the 13th International Workshop on RF Superconductivity, Beijing, China, 2007 (Ref. [2]), TUP69, pp. 304-307.

[25] F. Eozénou et al., in Proceedings of the 14th International Workshop on RF Superconductivity, Berlin, Germany, 2009 (Ref. [6]), THUPP070, pp. 781-785.

[26] A. Aspart et al., Physica C (Amsterdam) 441, 249 (2006); in the 12th International Workshop on $R F$ Superconductivity, 2005 Cornell University, Ithaca, NY, USA (Ref. [5]), pp. 455-458.

[27] N. Steinhau-Kühl et al., in the 12th International Workshop on RF Superconductivity, 2005 Cornell University, Ithaca, NY, USA (Ref. [5]), THPP085, pp. 464-466.

[28] T. Saeki et al., in 14th International Conference on RF Superconductivity (Ref. [4]), THPP085, pp. 824-828.

[29] F. Furuta and G. H. Hoffstaetter, in TTC meeting 2011, Beijing, China, see http://indico.ihep.ac.cn/getFile.py/ access ? contribId=54\&sessionId=8\&resId=2\&materialId= slides\&confId=2240. 